\title{
ROCK inhibitors alleviate myofibroblast transdifferentiation and vascular remodeling via decreasing TGF $\beta 1$-mediated RhoGDI expression
}

\author{
Jingjing Zhang ${ }^{1, \star}$, Lian Tang $^{1, \star}$, Fan Dai ${ }^{1}$, Yan $\mathrm{Qi}^{1}$, Lifeng Yang ${ }^{2}$, Zhaoguo Liu ${ }^{1}$, Li Deng ${ }^{3}$ \\ and Wenjuan $\mathrm{Yao}^{1}$ \\ ${ }^{1}$ Department of Pharmacology, School of Pharmacy, Nantong University, 19 QiXiu Road, Nantong, China \\ ${ }^{2}$ Department of Pharmacy, The First People's Hospital of Changzhou, Changzhou, China \\ ${ }^{3}$ Department of Pharmacy, Wuxi Children's Hospital, Wuxi, China
}

\begin{abstract}
The aim of this study was to investigate the effects of the Rho GDP dissociation inhibitor (RhoGDI) on TGF $\beta 1$-mediated vascular adventitia myofibroblast transdifferentiation and on the inhibition of ROCK inhibitors. Myofibroblast transdifferentiation and vascular remodeling model were induced by TGF $\beta 1$ in vitro and by balloon injury in vivo. H\&E (Hematoxylin \& Eosin) and PSR (Picrosirius Red) staining were used to observe vascular morphology while immunofluorescence, immunohistochemistry, and Western blotting were used to measure protein expression. Fasudil treatment reduced the expression of TGF $\beta 1$, RhoGDI1, and RhoGDI2 in addition to vascular remodeling in the rat balloon injury model. TGF $\beta 1$ induced the expression of $\alpha$-SMA, TGF $\beta R I$, phospho-TGF $\beta R I$, RhoGDI1, RhoGDI2, and collagen secretion in human aortic adventitial fibroblasts (HAAFs). These effects were diminished after treatment with Y27632. Suppressing both RhoGDI1 and RhoGDI2 expression also blocked TGF $\beta 1$-induced $\alpha$-SMA expression and collagen secretion in HAAFs. Moreover, TGF $\beta$ R inhibition blocked TGF $\beta 1$-mediated collagen secretion and the expression of a-SMA, RhoGDI1, and RhoGDI2. These data suggested that ROCK inhibitors alleviate myofibroblast transdifferentiation and vascular remodeling by decreasing TGF $\beta 1$-mediated expression of RhoGDI.
\end{abstract}

Key words: Vascular remodeling — Rho guanine nucleotide dissociation inhibitor - Rho-associated kinase - Myofibroblast - Transforming growth factor $\beta 1$

\section{Introduction}

Vascular remodeling that is characterized by intima-media thickening (IMT) is the pathological basis of various vascular diseases (Zhang et al. 2015). The adventitial layer that surrounds the blood vessels has long been considered a supporting tissue that provides the muscle layers with adequate nourishment (Sartore et al. 2001). However, adventitia also play an important role in the phenotypic conversion of vascular smooth muscle cells (VSMCs), resulting in neointima formation and vascular remodeling (Faggin et al. 1999; Li et al. 2000; Sartore et al. 2001). Adventitial fibroblasts in

\footnotetext{
* These authors contributed equally to this work.

Correspondence to: Wenjuan Yao, Department of Pharmacology, School of Pharmacy, Nantong University, 19 QiXiu Road, Nantong 226001, Jiangsu, China

E-mail: yaowenjuan0430@aliyun.com
}

experimental models of response to balloon vascular injury can be phenotypically converted into smooth muscle (SM)like cells termed myofibroblasts (Wilcox and Scott 1997; Zalewski and Shi 1997). Transforming growth factor $\beta 1$ (TGF $\beta 1$ ) is a potent driver of the differentiation of myofibroblast from fibroblasts and is accompanied by the expression of $\alpha$-smooth muscle actin ( $\alpha$-SMA) and collagen deposition (Hinz et al. 2007). The presence of stress fibers enables the generation of higher contractile forces by myofibroblasts compared to fibroblasts and the production of overt migratory and proliferative activities in subendothelial spaces (Dunkern et al. 2007; Sun et al. 2016). Recent studies have indicated that the MEK and Smad2/3-p38MAPK-ERK1/2 pathways participate in TGF- $\beta 1$-induced myofibroblast transdifferentiation in human Tenon fibroblasts (Lin et al. 2018; Wen et al. 2019). In addition, connective tissue growth factor (CTGF) is an essential downstream mediator in the TGF- $\beta 1$-induced transdifferentiation of myofibroblasts 
from Graves' orbital fibroblasts (Tsai et al. 2018). However, the molecular mechanism underlying TGF $\beta 1$-induced myofibroblast transdifferentiation in vascular adventitial fibroblasts remains unclear.

The Rho-specific guanine nucleotide dissociation inhibitor (RhoGDI) is critical for homeostasis of Rho proteins and crosstalk between Rho protein family members (Boulter and Garcia-Mata 2010). Depletion of RhoGDI1, or its yeast ortholog RDI1, can result in almost complete degradation of RhoA, Rac1, and Cdc42 proteins by proteasomes in eukaryotic cells (Boulter et al. 2010). Furthermore, numerous studies have shown that RhoGDI expression levels are associated with the presence of several cancers (Zhao et al. 2008). For example, RhoGDI1 expression is upregulated in colorectal and ovarian cancer cells, wherein high expression levels correlate with the increased invasion of cancerous cells and resistance to chemotherapy (Jones et al. 2002; Zhao et al. 2010). In contrast, RhoGDI1 expression is reduced in brain cancers and correlates with reduced expression of RhoA and RhoB, but not Rac1 proteins (Forget et al. 2002). The expression levels of RhoGDI2 also highly vary among cancers (Harding and Theodorescu 2007). However, little is known of the physiological function of RhoGDI and how RhoGDI expression is regulated. Fasudil is a Rho-associated kinase (ROCK) inhibitor that has been clinically applied and significantly contributes to the treatment of cardiovascular, neurological, and oncologic diseases (Kishi et al. 2005). Y27632 is another common ROCK inhibitor that has been extensively investigated in experimental studies (Pan et al. 2013). The inhibition of ROCK signaling pathways is associated with numerous beneficial influences in the treatment of different diseases in both research and clinical setting (Pan et al. 2013).

In the present study, an in vivo balloon injury-induced vascular remodeling model and an in vitro TGF $\beta 1$-induced myofibroblast transdifferentiation model were used to investigate the effects of ROCK inhibitors on TGF $\beta 1$-mediated RhoGDI expression, fibroblast phenotypic modulation, and vascular remodeling.

\section{Materials and Methods}

\section{Materials}

Recombinant human TGF $\beta 1$ was obtained from Novoprotein (\#CA59; Shanghai, China), the TGF $\beta$ receptor inhibitor LY2109761 from Chemcatch (CC2860; Irvine, CA, USA), and the ROCK inhibitor Y27632 from Selleckchem (\#129830-382; Houston, TX, USA). Fasudil was purchased from the Yuan Ye Biological Technology Co., Ltd. (\#105628-07-7; Shanghai, China). A Fogarty $2 \mathrm{~F}$ balloon catheter was purchased from the Baxter Health Care Corporation (\#12TLW804 F; Irvine, CA, USA). A Sirius Red staining solution kit was sourced from Solarbio (G1470; Beijing, China), while a Sirius Red collagen detection kit was purchased from Chondrex Inc. (\#9062; Washington, USA). A DyLight 488-SABC SP kit was purchased from BosterBio (SA1094; Wuhan, China). A total RNA purification kit (\#TR01), RevertAid first strand cDNA synthesis kit (\#K1622), and DreamTaq PCR master mix (\#K1071) were all purchased from Thermo Scientific (Shanghai, China). In addition, siRNAs and PCR primers were purchased from Biomics Biotechnologies (Nantong, China). RIPA lysis buffer (CW2333S) and a BCA protein assay kit (CW0014S) were both purchased from CWbio (Beijing, China), while an SDS-PAGE Gel Quick Preparation kit was sourced from Beyotime Biotechnology (P0012AC; Nantong, China). A dual-color, pre-stained protein marker was obtained from Epizyme Biological Technology (WJ101; Shanghai, China) and anti-TGF $\beta 1$ (A2124) and -RhoGDI1 (A1214) antibodies were obtained from ABclonal Technology (Wuhan, China). The antibody against phospho-TGF $\beta R I$ was purchased from Sabbiotech (\#12388; Maryland, USA), while the antibodies against TGFßRI (ab31013), a-SMA (ab124964), and RhoGDI2 (ab181252) were purchased from Abcam Co. (Cambridge, UK). The primary antibody against GAPDH (\#5174) was obtained from Cell Signaling Technology (Beverly, MA, USA). HRP-conjugated AffiniPure Goat Anti-Rabbit IgG $(\mathrm{H}+\mathrm{L})$ was purchased from Proteintech (SA00001-2; Chicago, IL, USA) and Fluorescein (FITC)-conjugated AffiniPure Donkey Anti-Rabbit IgG (H $+\mathrm{L}$ ) was purchased from BBI Life Sciences (D110051; Hong Kong, China). An SABC immunohistochemistry staining kit was sourced from BosterBio (SA1028; Wuhan, China). Lastly, fibroblast medium-2 (FM-2) (\#2331) was purchased from ScienCell Research Laboratories (CA, USA). All other chemicals that were used in this study were of analytical grade and sourced from China.

\section{Cell cultures and treatment}

Human aortic adventitial fibroblasts (HAAFs) were purchased from ScienCell Research Laboratories (Catalog \#6120; CA, USA). The cells were cultured in FM-2 medium at $37^{\circ} \mathrm{C}$ in a humidified incubator with a $5 \% \mathrm{CO}_{2}$ atmosphere with replacement of culture medium every three days. Cells were collected and used for experimentation from the third through seventh passages. HAAFs were first pretreated with $20 \mu \mathrm{M}$ Y 27632 for $30 \mathrm{~min}$ or $10 \mu \mathrm{M}$ LY2109761 for $24 \mathrm{~h}$ and then exposed to $10 \mathrm{ng} / \mathrm{ml}$ TGF $\beta 1$ for $72 \mathrm{~h}$.

\section{Rat carotid artery balloon injury model}

All animal experiments were performed with male SpragueDawley rats that were obtained from the Animal Center of Nantong University (Nantong, China) and aged 42-49 days with weights $\sim 250 \mathrm{~g}$. All animal experimental proce- 
dures conformed to the NIH Guide for the Care and Use of Laboratory Animals and were approved by the Ethics Committee and the Animal Care and Use Committee of Nantong University. All animals were housed under $12 \mathrm{~h} \mathrm{light} /$ dark cycles at $20^{\circ} \mathrm{C}$ with a humidity of $75 \%$ and received a normal diet with water ad libitum. Ten male rats were randomly assigned into each of three groups $(n=10 /$ group): sham-operation, model group (balloon injury), and the fasudil-treated groups. Rat carotid artery balloon injury was established as described previously (Tulis 2007). Briefly, rats were anesthetized by an intraperitoneal injection of $4 \%$ pentobarbital, and a $2 \mathrm{~F}$ Fogarty balloon embolectomy catheter was introduced into the carotid artery and passed up to the aortic arch after opening of the vessel. The balloon was then inflated to distend the common carotid artery and withdrawn with rotation. Fasudil was dissolved in normal saline solution and injected intraperitoneally $(30 \mathrm{mg} / \mathrm{kg} / \mathrm{d})$ starting the day after the operation and continuing daily for 14 days. After 14 days, the balloon-injured segment of the artery was removed, washed in saline solution on ice, and then used in subsequent experiments.

\section{siRNA transfection}

RhoGDI1 and RhoGDI2 siRNAs were used with the following sequence structure: siRhoGDI1 (sense: 5'-GUGUGGAGUACCGGAUAAAdTdT-3'; antisense: 5'-UUUAUCCGGUACUCCACACdTdT-3'), siRhoGDI2 (sense: 5'-CACAAGAGAACAAGAAUAAdTdT-3'; antisense: 5'-UUAUUCUUGUUCUCUUGUGdTdT-3'). The Lipofectamine 2000 reagent and synthetic siRNAs $(20 \mu \mathrm{M}$ in DEPC water) were diluted using Opti-MEM and then incubated at room temperature for $20 \mathrm{~min}$. The mixture was added to cells, followed by incubation for $48 \mathrm{~h}$. Successful interference with the target gene was then confirmed using Western blot analyses. The siRNA transfected cells were subsequently treated with $10 \mathrm{ng} / \mathrm{ml}$ TGF $\beta 1$ for $72 \mathrm{~h}$.

\section{H\&E staining and Picrosirius Red (PSR) staining}

The injured segments of the left common carotid arteries were isolated, fixed in $4 \%$ paraformaldehyde, and embedded in optimal cutting temperature compound (OCT). The OCT-embedded $5-\mu \mathrm{m}$ sections were then stained with hematoxylin aqueous solution for $5 \mathrm{~min}$, differentiated with hydrochloric acid for $30 \mathrm{~s}$, and stained again with eosin for $2 \mathrm{~min}$. The sections were then dehydrated using ethanol and cleared using xylene. The intimal area was calculated as the area of the internal elastic lamina after subtracting the luminal area. The medial area was calculated as the external elastic lamina area after subtracting the area of the internal elastic lamina area. The sections were then stained with hematoxylin aqueous solution for 10-20 min, differentiated with hydrochloric acid for $30 \mathrm{~s}$, and again stained with Sirius Red solution for $1 \mathrm{~h}$. The red-stained collagen was then qualified by staining intensity. All microscopy images were captured using an Olympus digital camera (Olympus, Tokyo, Japan) and analyzed using the Image-Pro Plus software program (Media Cybernetics, Rockville, MD, USA).

\section{Picrosirius Red collagen detection}

Total collagen (types I to V) content in the medium was measured using a Sirius Red collagen detection kit according to the manufacturer's instructions. Briefly, a $1 \times$ acetic acid solution was used to prepare standards and samples. A $100 \mu \mathrm{l}$ volume of blanks, standards, and samples were added to $1.5 \mathrm{ml}$ centrifuge tubes and then mixed with $500 \mu \mathrm{l}$ of Sirius Red solution for $20 \mathrm{~min}$ at room temperature. All assays were performed in duplicate. The tubes were then centrifuged at 10, $000 \mathrm{rpm}$ for $3 \mathrm{~min}$, followed by removal of the supernatants. Pellets were washed three times with $500 \mu \mathrm{l}$ of washing solution and then dissolved in $250 \mu \mathrm{l}$ of extraction buffer. The solutions were transferred to 96 -well plates and their absorbance was measured at $530 \mathrm{~nm}$ using an ELISA plate reader (BioTek Instruments, Vermont, USA).

\section{Immunofluorescence staining}

The 5- $\mu \mathrm{m}$ sections were fixed with $4 \%$ paraformaldehyde and incubated at room temperature for $60 \mathrm{~min}$, followed by quenching with $5 \% \mathrm{BSA}$ blocking buffer at $37^{\circ} \mathrm{C}$ for $30 \mathrm{~min}$, and incubation with primary antibodies overnight at $4^{\circ} \mathrm{C}$. After washing three times with PBS, the sections were incubated with anti-rabbit IgG for an additional $30 \mathrm{~min}$ at $37^{\circ} \mathrm{C}$. Staining was then visualized using a fluorescence microscope (Olympus BX51) after DAPI staining of tissues for $20 \mathrm{~min}$. Imaging was performed using an Olympus UPlanSApo 20x / 0.75 objective. Hardware settings were maintained at the same levels for all experiments (FITC channel: laser power 60 , exposure time $80 \mathrm{~ms}$ ). Fluorescence intensity was quantified using the Image-Pro Plus software program. Specifically, quantification of fluorescence was calculated after conversion of the standardized pictures taken with the fluorescence microscope into 8-bit gray-scale images.

\section{Immunohistochemistry}

Immunohistochemical staining against TGF $\beta 1$ and a-SMA were performed using a strept avidin-biotin complex (SABC) immunohistochemistry staining kit and following the manufacturer's instructions. HAAFs grown on glass coverslips were washed with PBS and fixed with 4\% paraformaldehyde for 20 $\mathrm{min}$. The sections were allowed to equilibrate to room temperature and were then fixed with $4 \%$ paraformaldehyde for $60 \mathrm{~min}$. The cells or sections were then incubated with a mix- 
ture of $30 \% \mathrm{H}_{2} \mathrm{O}_{2}$ and methanol for $15 \mathrm{~min}$ and blocked for $30 \mathrm{~min}$ at room temperature with PBS buffer containing 5\% BSA buffer. After overnight incubation at $4^{\circ} \mathrm{C}$ with primary antibodies, the samples were incubated with biotinylated anti-rabbit IgG for $2 \mathrm{~h}$ and SABC for $1 \mathrm{~h}$. The samples were then visualized using a diaminobenzidine (DAB) staining kit followed by counterstaining with hematoxylin in order to stain the target proteins brown. All images were captured using an Olympus digital camera (Olympus, Tokyo, Japan) and analyzed using the Image-Pro Plus software program (Media Cybernetics, Rockville, MD, USA).

\section{Western blot}

To conduct Western blot analyses, cells were washed with PBS and transferred to microcentrifuge tubes on ice. Then, 30-40 $\mu$ l of lysis buffer (RIPA, 1 mM PMSF) was added to the cell solutions. After incubation for $40 \mathrm{~min}$, the lysates were centrifuged at 12, $000 \mathrm{rpm}$ for $15 \mathrm{~min}$ and at $4^{\circ} \mathrm{C}$. Stainless steel beads were then added to the arteries for tissue protein extraction, followed by the addition of $160 \mu$ of lysis buffer (RIPA, $1 \mathrm{mM}$ PMSF). Incubation on the ice was conducted for $40 \mathrm{~min}$, followed by centrifugation at $12000 \mathrm{r} / \mathrm{min}$ for $15 \mathrm{~min}$ at $4^{\circ} \mathrm{C}$. Protein concentrations were then measured using Bradford assays. The protein samples were then separated by SDS-PAGE (polyacrylamide gel electrophoresis) and transferred to nitrocellulose membranes. The membranes were blocked for $2 \mathrm{~h}$ at room temperature with 5\% nonfat milk in TBS (pH 7.4) containing 0.1\% Tween-20. After blocking, samples were incubated with different primary antibodies overnight at $4^{\circ} \mathrm{C}$ and HRP-conjugated secondary antibodies for $2 \mathrm{~h}$ at room temperature. The blots were then visualized with an ECL detection system (Amersham Biosciences) using GAPDH as an internal standard. Relative signal intensities of the signals were quantified using densitometry and Imaging software from Labworks.

\section{Statistical analysis}

All data are presented as means \pm SD. Statistical significance of differences were evaluated by one-way analyses of variance (ANOVA) followed by Tukey's post-hoc tests in the SPSS 22.0 program. Statistical significance was considered as $p<0.05$.

\section{Results}

ROCK inhibitors reduce vascular remodeling and myofibroblast transdifferentiation by decreasing TGF $\beta 1$ expression and TGF $\beta R I$ activation

The effects of fasudil on vascular remodeling were evaluated in vivo using a rat balloon injury model. Balloon in- jury resulted in obvious vascular remodeling and collagen production, as evinced by $\mathrm{H} \& \mathrm{E}$ and PSR staining, intima/ media area ratios $(0.17 \pm 0.03 v s .0 .33 \pm 0.06, p<0.05)$, and higher collagen contents $(28.24 \pm 3.10 v s .89 .52 \pm 10.11, p<$ 0.01 ) (Fig. 1A). In particular, fasudil treatment significantly reduced neointima formation and collagen secretion, as indicated by a $36 \%$ decrease in the intima/media area ratio and a $66 \%$ estimated reduction in collagen content. These results suggest that ROCK inhibition can effectively reverse vascular remodeling and collagen production. Further, immunohistochemistry and Western blot assays indicated that treatment with fasudil reduced TGF $\beta 1$ expression in rat carotid arteries after balloon injury (Figs. $1 \mathrm{~B}$ and $\mathrm{C}$ ). TGF $\beta 1$ is one of the most important regulators involved in the pathogenesis of cardiovascular diseases like restenosis following angioplasty and atherosclerosis (Verrecchia and Mauviel 2002). Consequently, the effects of ROCK inhibition on TGF $\beta 1$-induced myofibroblast transdifferentiation were evaluated in vitro. TGF $\beta 1$ significantly induced expression of $\alpha$-SMA in HAAF cells and the secretion of collagen into medium (Figs. 1D, E and F), while these effects were inhibited by $Y 27632$ treatment. The inhibition of TGF $\beta 1$-induced SMA expression by $\mathrm{Y} 27632$ is consistent with previous investigations (Ji et al. 2014). Furthermore, Y27632 treatment dramatically decreased TGF $\beta 1$-induced expression and phosphorylation of TGF $\beta$ RI (Fig. 1G). Together, these data suggested that ROCK inhibition may reduce myofibroblast transdifferentiation and vascular remodeling by suppressing TGF $\beta 1 /$ TGF $\beta$ RI activation.

\section{ROCK inhibitors decrease RhoGDI expression in TGF $\beta 1$ -} -induced HAAFs and balloon injury models

To clarify the role of RhoGDI in myofibroblast transdifferentiation in response to TGF $\beta 1$, the expression of both RhoGDI1 and RhoGDI2 was evaluated using Western blot analyses. RhoGDI1 and RhoGDI2 expression was significantly elevated by TGF $\beta 1$ treatment compared to the control group (Fig. 2A). In contrast, Y27632 treatment significantly decreased both RhoGDI1 and RhoGDI2 expression in TGF $\beta 1$-treated HAAFs. Further, immunostaining assay and Western blot analyses demonstrated that treatment with fasudil reduced RhoGDI1 and RhoGDI2 expression in rat carotid arteries after balloon injures (Figs. $2 \mathrm{~B}$ and C). These results are consistent with those of the in vitro investigation and suggest that ROCK inhibition may reduce vascular remodeling by inhibiting RhoGDI expression.

\section{The TGF $\beta$ receptor participates in TGF $\beta 1$-induced RhoGDI} expression and myofibroblast transdifferentiation

To further elucidate the role of RhoGDI in TGF $\beta 1$-mediated myofibroblast transdifferentiation, the expression of RhoGD 
was suppressed using siRNA. Suppression of both RhoGDI1 and RhoGDI2 expression significantly inhibited TGF $\beta 1$ induced $\alpha$-SMA expression and collagen secretion (Figs. $3 \mathrm{~A}-\mathrm{E})$, suggesting that RhoGDI was indeed involved in
TGF $\beta 1$-mediated myofibroblast transdifferentiation. To assess whether TGF $\beta 1$ stimulates RhoGDI expression via its receptor, cells were incubated with the TGF $\beta$ receptor inhibitor LY2109761, and RhoGDI expression was measured. The
A
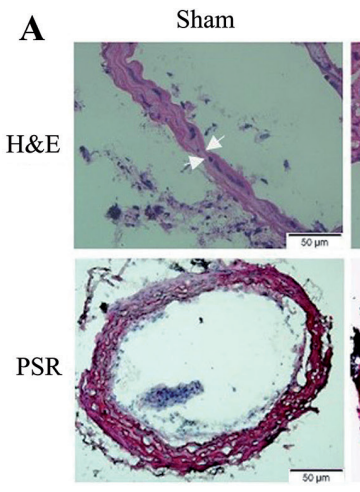

B

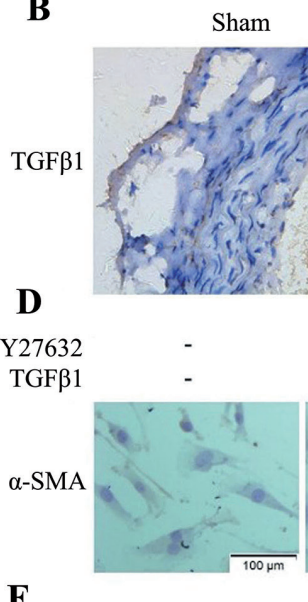

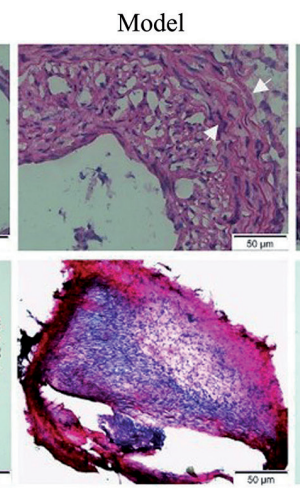

Model

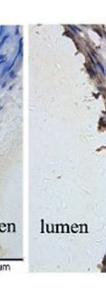

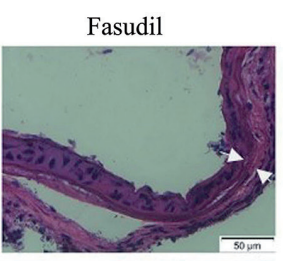

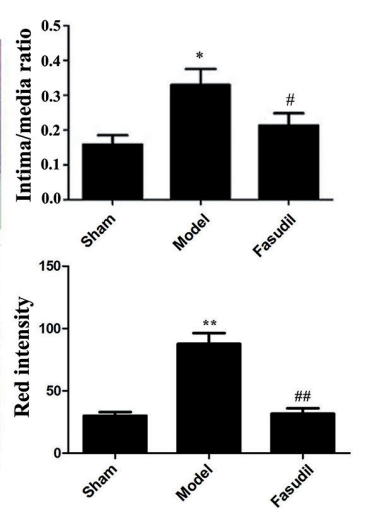

C

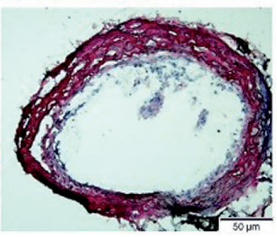

Fasudil
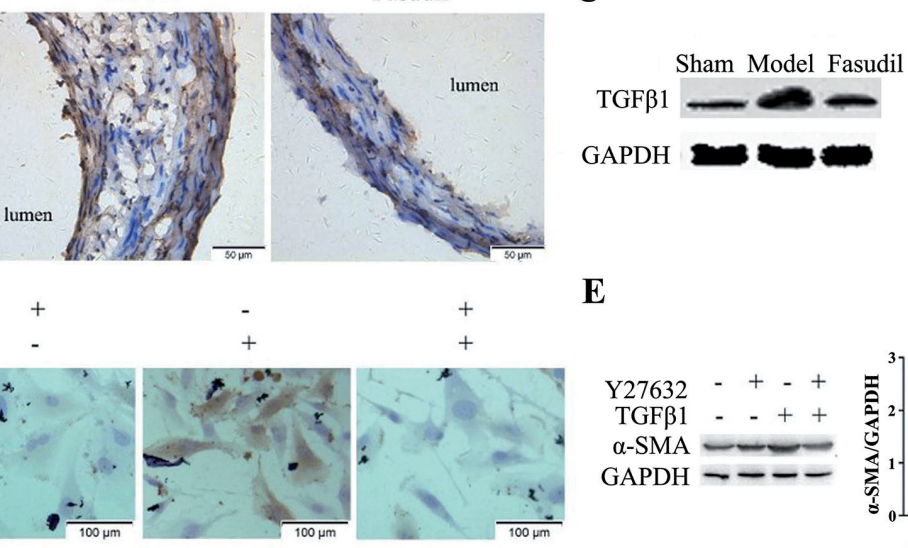

GAPDH

$\mathbf{E}$
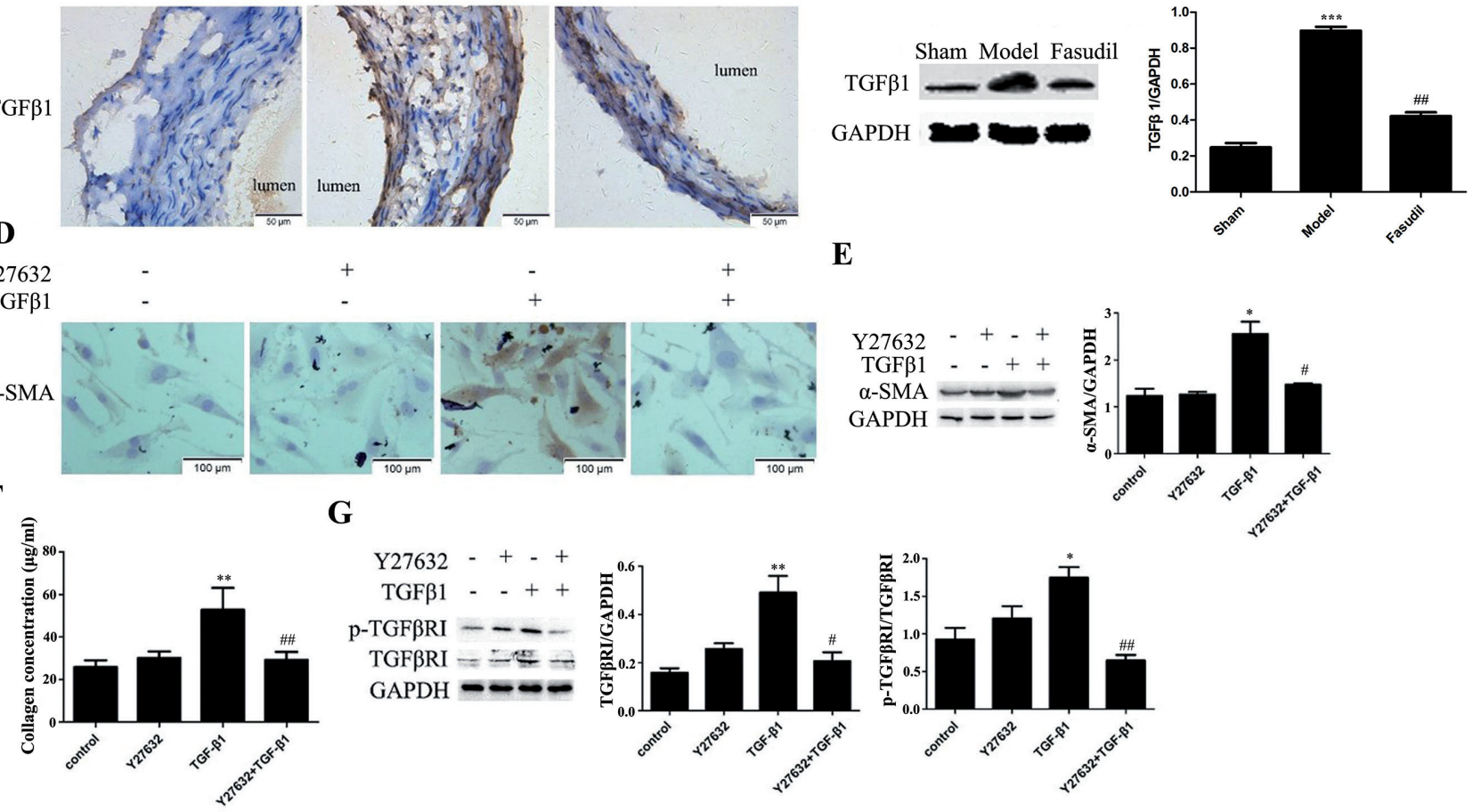

$\mathbf{G}$
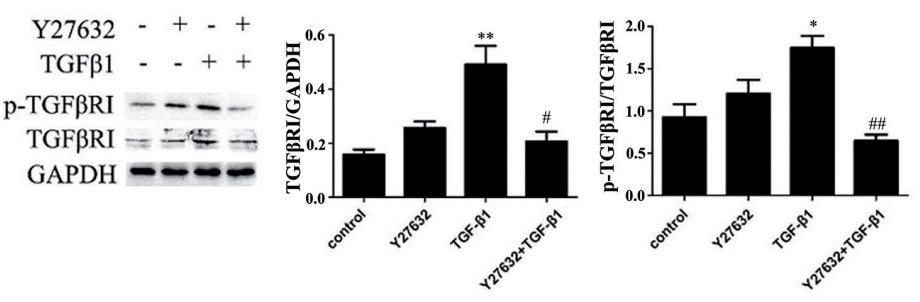

Figure 1. The effects of ROCK inhibitors on vascular remodeling and myofibroblast transdifferentiation. A. H\&E and PSR staining of arteries 14 days after balloon injury. Rats without balloon injury were used as the sham treatment group. Arrows indicate internal and external elastic lamina, while histograms show the intima-media area ratios and the collagen (red stained) intensity. ${ }^{*} p<0.05$ and ${ }^{* *} p<0.01 v s$. the sham treatment group; ${ }^{\#} p<0.05$ and ${ }^{\# \#} p<0.01$ vs. the injury model group $(n=10)$. B. Immunohistochemistry staining of TGF $\beta 1$, wherein positive staining is indicated by brown coloration and nuclei were stained with hematoxylin in blue. C. Western blot showing TGF $\beta 1$ expression. Histogram shows the ratio of TGF $\beta 1$ to GAPDH. ${ }^{* *} p<0.001 v$ s. the sham operation group; ${ }^{\# \#} p<0.01 v s$. the injury model group ( $\left.n=10\right)$. D. Immunohistochemistry staining of $\alpha$-SMA in HAAFs. Cells were pretreated with $20 \mu \mathrm{M}$ Y 27632 for 30 min and then exposed to $10 \mathrm{ng} /$ $\mathrm{ml}$ TGF $\beta 1$ for an additional $72 \mathrm{~h}$. Untreated cells were used as controls. $\alpha$-SMA-positive cells are indicated by brown coloration. E. Western blot showing the expression of $\alpha$-SMA. Histogram shows the ratio of $\alpha$-SMA to GAPDH. ${ }^{*} p<0.05 v s$. the control group; ${ }^{\#} p<0.05 v s$. the TGF $\beta 1$-treated group $(n=3)$. F. Detection of collagen secretion into media. Histogram shows collagen concentrations in each group based on absorbance at $530 \mathrm{~nm} .{ }^{* *} p<0.01 v$ s. the control group; ${ }^{\# \#} p<0.01 v s$. the TGF $\beta 1$-treated group $(n=3)$. G. Western blot showing the expression of TGF $\beta$ RI and phospho-TGF $\beta$ RI. Histograms show the ratio of expression levels of phospho-TGF $\beta R I$ to TGF $\beta$ RI or TGF $\beta R I$ to GAPDH. ${ }^{\star} p<0.05,{ }^{* *} p<0.01 v$ s. the control group; ${ }^{\#} p<0.05$ and ${ }^{\# \#} p<0.01 v s$. the TGF $\beta 1$-treated group $(n=3)$. (See online version for color figure.) 
expression levels of RhoGDI1 and RhoGDI2 were significantly decreased after LY2109761 incubation in the TGF $\beta 1$ treatment group (Fig. 3F), suggesting that TGF $\beta 1$ stimulates RhoGDI expression through TGF $\beta$ receptor activation. The effects of TGF $\beta$ receptor inhibition on TGF $\beta 1$-induced cell transdifferentiation were also evaluated and revealed that LY2109761 pretreatment clearly suppressed TGF $\beta 1$-induced a-SMA expression and collagen secretion (Figs. 3G-I).



B

RhoGDI1
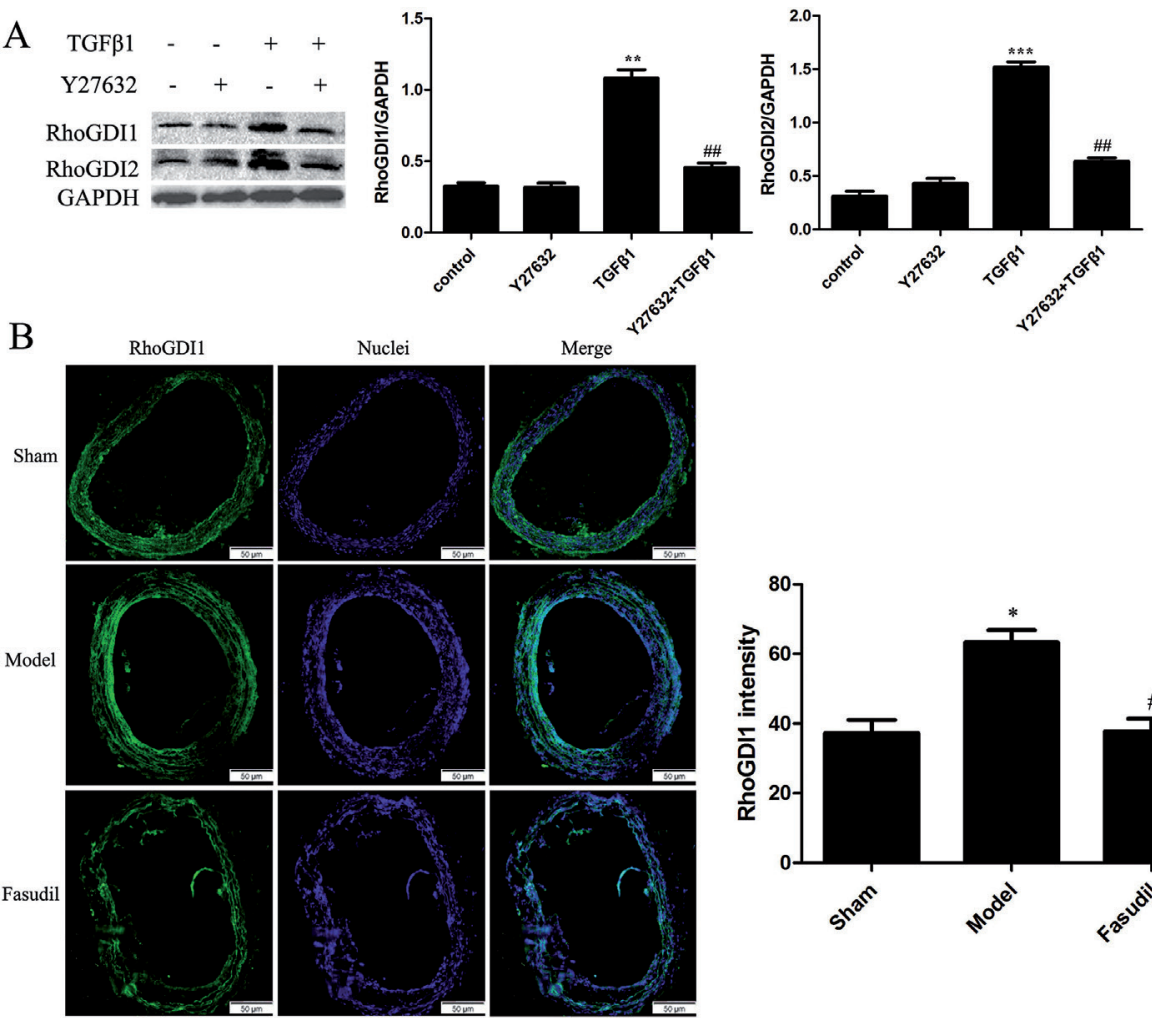

Nuclei Merge
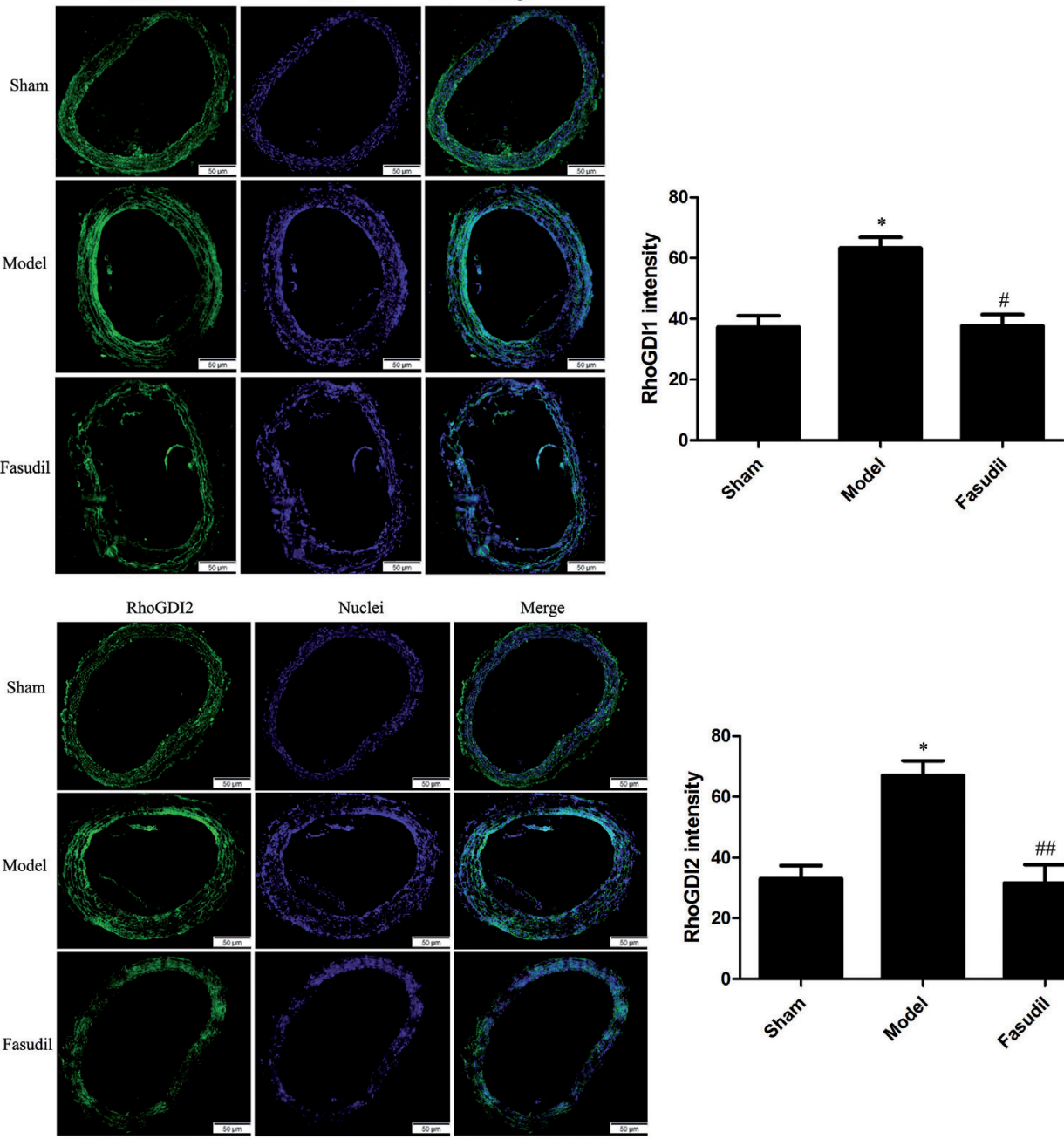

c

Sham Model Fasudil

RhoGDI1

RhoGDI2

GAPDH
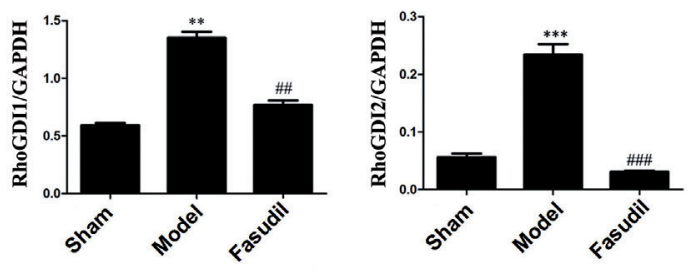

Figure 2. The effect of ROCK inhibition on RhoGDI expression. A. Western blot showing the expression of RhoGDI1 and RhoGDI2. Cells were pretreated with $20 \mu \mathrm{M}$ Y 27632 for $30 \mathrm{~min}$ and then exposed to $10 \mathrm{ng} /$ $\mathrm{ml}$ TGF $\beta 1$ for an additional $72 \mathrm{~h}$. Untreated cells were used as the control group. Histograms show the ratio of RhoGDI1 or RhoGDI2 to GAPDH. ${ }^{* *} p<0.01$ and ${ }^{* * *} p<0.001 v s$. the control group; ${ }^{\# \#} p<0.01$ vs. the TGF $\beta 1$-treated group ( $n$ $=3$ ). B. Immunofluorescence staining of RhoGDI1 and RhoGDI2 (shown in green), with nuclei stained with DAPI in blue. Histograms show the fluorescence intensity of staining. Rats without balloon injury were used as the sham operation group. ${ }^{\star} p<0.05$ vs. the sham operation group; ${ }^{\#} p<$ 0.05 and ${ }^{\# \#} p<0.01$ vs. the injury model group $(n=10)$. C. Western blot showing RhoGDI1 and RhoGDI2 expression after balloon injury. Rats without balloon injury were used as the sham operation group. Histograms show the ratio of RhoGDI1 or RhoGDI2 to GAPDH. ${ }^{* *} p<0.01$ and ${ }^{* * *} p<0.001$ vs. the sham operation group; ${ }^{\# \#} p<0.01$ and ${ }^{\# \#} p<0.001 v s$. the injury model group $(n=10)$. (See online version for color figure.) 


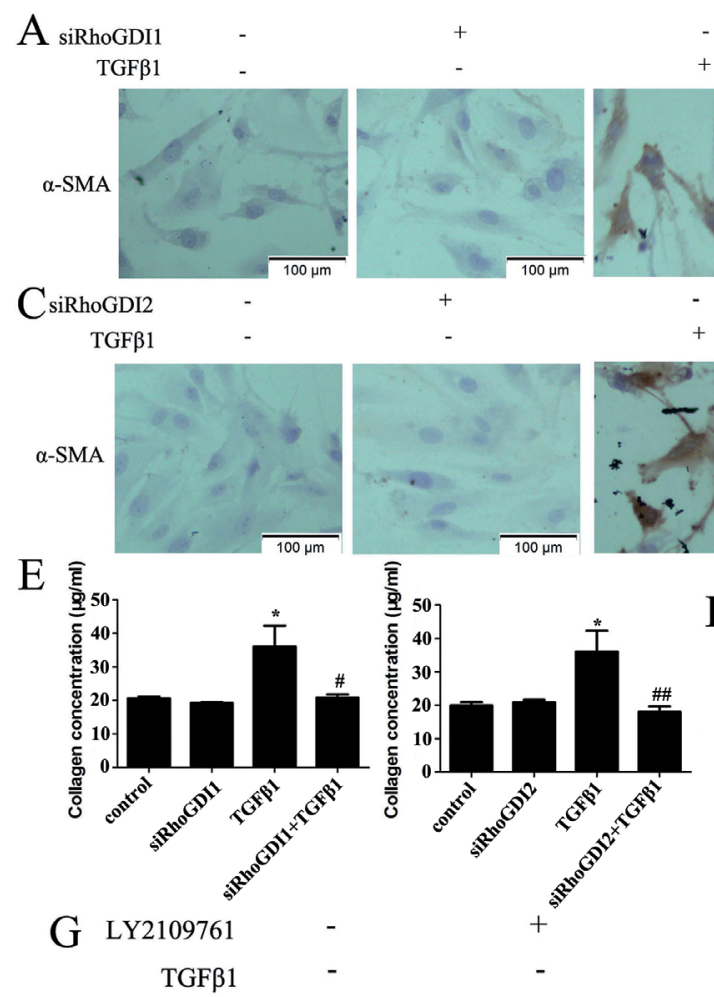

$\begin{array}{ll}- & + \\ + & +\end{array}$



$+$

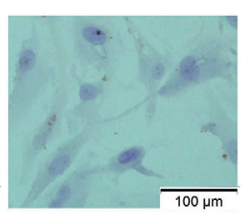

D
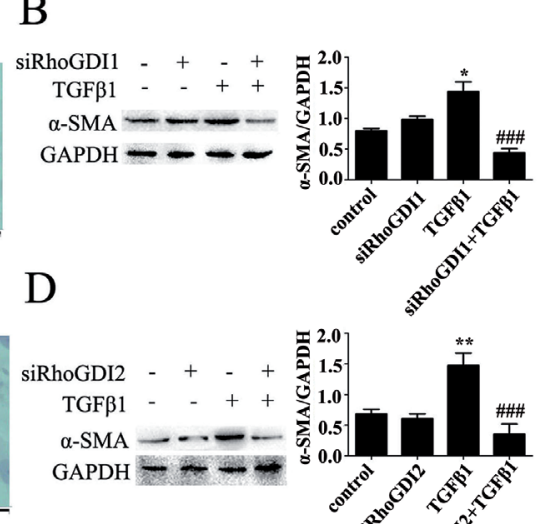

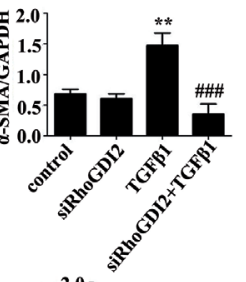

F


Figure 3. A. The effect of RhoGDI1 suppression on TGF $\beta 1$ induced $\alpha$-SMA expression using immunohistochemistry staining. Cells were treated with $10 \mathrm{ng} / \mathrm{ml}$ TGF $\beta 1$ for $72 \mathrm{~h}$ and nuclei were stained with hematoxylin and are indicated in blue. B. Western blot showing the expression of a-SMA. Cells were treated with $10 \mathrm{ng} / \mathrm{ml}$ TGF $\beta 1$ for $72 \mathrm{~h}$ and untreated cells were used as the control group. Histogram shows the ratio of $\alpha$-SMA to GAPDH. ${ }^{*} p<0.05 v s$. the control group;

$\# \# p<0.001 v$ s. the TGF $\beta 1$-treated group $(n=3)$. C. The effect of RhoGDI2 suppression on TGF $\beta 1$-induced $\alpha$-SMA expression using immunohistochemistry staining. Nuclei were stained with hematoxylin and are indicated in blue. D. Western blot showing a-SMA expression. Cells were treated with $10 \mathrm{ng} / \mathrm{ml}$ TGF $\beta 1$ for $72 \mathrm{~h}$ and untreated cells were used as the control group. Histogram shows the ratio of $\alpha$-SMA to GAPDH. ${ }^{* *} p<0.01 v$ s. the control group; ${ }^{\# \#} p<0.001 v s$. the TGF $\beta 1$-treated group $(n=3)$. E. The effect of RhoGDI suppression on TGF $\beta 1$-induced collagen secretion using Sirius Red Collagen Detection Kit. Histograms show the concentrations of collagen in each group, indicated by absorbance values at $530 \mathrm{~nm}$. Untreated cells were used as the control group. ${ }^{*} p<0.05$ $v s$. the control group; ${ }^{\#} p<0.05$ and ${ }^{\# \#} p<0.01 v s$. the TGF $\beta 1$-treated group $(n=3)$. F. The effect of LY2109761 pretreatment on the expression of RhoGDI. Cells were pretreated with $10 \mu \mathrm{M}$ LY2109761 for $24 \mathrm{~h}$ and then exposed to $10 \mathrm{ng} / \mathrm{ml} \mathrm{TGF} \beta 1$ for $72 \mathrm{~h}$. Cells without treatment was used as the control group. Histograms show the ratio of RhoGDI1 or RhoGDI2 to GAPDH. ${ }^{\star} p<0.05$ and ${ }^{* *} p<0.01 v$ s. the control group; ${ }^{\#} p<0.05$ and ${ }^{\# \#} p<0.01 v s$. the TGF $\beta 1$-treated group $(n=3)$. G. Immunohistochemistry determination of $\alpha$-SMA expression. Nuclei were stained with hematoxylin and are shown in blue. H. Western blot showing $\alpha$-SMA expression. Histogram shows the ratio of $\alpha$-SMA to GAPDH. ${ }^{* *} p<0.01 v$ s. the control group; ${ }^{\# \#} p<0.01 v s$. the TGF $\beta 1$-treated group $(n=3)$. I. The effect of LY2109761 pretreatment on TGF $\beta 1$-induced collagen secretion. Histogram shows the concentrations of collagen in each group. Untreated cells were used as the control group. ${ }^{\star} p<0.05 v$ s. the control group; ${ }^{\#} p<0.05 v s$. the TGF $\beta 1$-treated group ( $n$ =3). (See online version for color figure.) 


\section{Discussion}

Vascular remodeling is a common pathophysiological process following cardiovascular diseases like atherosclerosis and hypertension. Consequently, investigation of myofibroblast transdifferentiation in vascular adventitial fibroblasts has become increasingly important for understanding vascular remodeling mechanisms. RhoGDIs are important physiological regulators that belong to the Rho family of small GTPases. An emerging body of literature suggests that their activity is altered during carcinogenesis and tumor progression (Boulter et al. 2010). However, the effects of RhoGDI on myofibroblast phenotypic modulation and vascular remodeling are not well understood. Indeed, this is the first study to report that RhoGDI participates in TGF $\beta 1$-mediated myofibroblast transdifferentiation and vascular remodeling, and also the first to investigate the inhibitory effects of ROCK inhibitors.

Our results demonstrated that TGF $\beta 1$ promotes RhoGDI1 and RhoGDI2 expression by activating the TGF $\beta$ receptor in TGF $\beta 1$-induced HAAF cells in vitro and via the same pathway in an in vivo balloon injury model. In addition, the suppression of RhoGDI and inhibition of the TGF $\beta$ receptor significantly decreased TGF $\beta 1$-induced $\alpha$-SMA expression and collagen secretion in HAAFs. These observations suggested that RhoGDI affects the phenotypic modulation of TGF $\beta 1$-induced myofibroblast via the TGF $\beta$ receptor. ROCK Inhibition has been previously shown to exhibit therapeutic benefits for a variety of diseases. Y27632 and fasudil are the most well-known ROCK inhibitors, and have been used extensively in previous experiments (Pan et al. 2013). Here, fasudil treatment resulted in decreases in the expression of TGF $\beta 1$, neointima formation, and collagen production in an in vivo rat balloon injury model. In addition, Y27632 treatment inhibited TGF $\beta 1$-mediated myofibroblast transdifferentiation and the expression and activation of TGF $\beta$ receptors. These results suggested that ROCK inhibitors reduce myofibroblast transdifferentiation and vascular remodeling by regulating TGF $\beta 1$ and its downstream pathways. Moreover, ROCK inhibition reduced levels of both RhoGDI1 and RhoGDI2 in an in vivo balloon injury model and an in vitro model of TGF $\beta 1$-induced HAAF differentiation. In summary, the primary findings of this study are that: (1) RhoGDI expression is involved in regulating myofibroblast phenotypic modula-

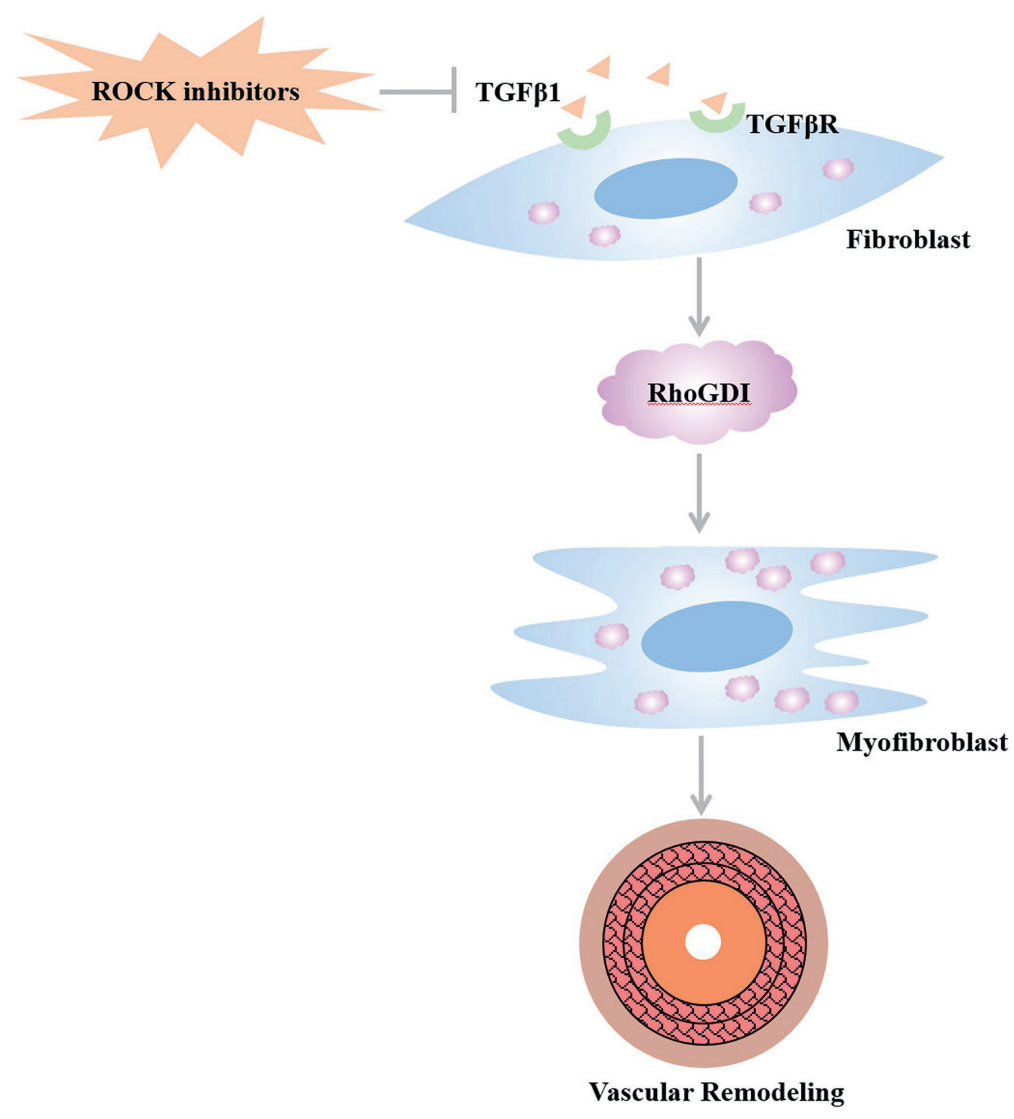

Figure 4. Diagram of the effects of ROCK inhibitors on TGF $\beta 1$-mediated RhoGDI expression, myofibroblast transdifferentiation and vascular remodeling. 
tion and vascular remodeling as mediated by TGF $\beta 1$ and its receptor; and (2) ROCK inhibitors inhibit TGF $\beta 1$-induced myofibroblast transdifferentiation and vascular remodeling by regulating the activity of the TGF $\beta 1 /$ RhoGDI pathway (Fig. 4).

Conflict of interest. The authors have no conflicts of interest to declare in relation to this article.

Acknowledgments. This work was supported by grants from the National Natures Science Foundation of Nantong City (No. JC2018059), the Jiangsu Planned Projects for Postdoctoral Research Funds (No. 1601107C) and the National Natural Science Foundation of China (No. 81703884).

\section{References}

Boulter E, Garcia-Mata R, Guilluy C, Dubash A, Rossi G, Brennwald PJ, Burridge K (2010): Regulation of Rho GTPase crosstalk, degradation and activity by RhoGDI1. Nat. Cell. Biol. 12, 477-483 https://doi.org/10.1038/ncb2049

Boulter E, Garcia-Mata R (2010): RhoGDI: A rheostat for the Rho switch. Small GTPases 1, 65-68 https://doi.org/10.4161/sgtp.1.1.12990

Dunkern TR, Feurstein D, Rossi GA, Sabatini F, Anathematize A (2007): Inhibition of TGF- $\beta$ induced lung fibroblast to myofibroblast conversion by phosphodiesterase inhibiting drugs and activators of soluble guanylyl cyclase. Eur. J. Pharmacol. 572, 12-22 https://doi.org/10.1016/j.ejphar.2007.06.036

Faggin E, Puato M, Zardo L, Franch R, Millino C, Sarinella F, Pauletto P, Sartore S, Chiavegato A (1999): Smooth musclespecific SM22 protein is expressed in the adventitial cells of the balloon-injured rabbit carotid artery. Arterioscler. Thromb. Vasc. Biol. 19, 1393-1404 https://doi.org/10.1161/01.ATV.19.6.1393

Forget MA, Desrosiers RR, Del M, Moumdjian R, Shedid D, Berthelet F, Beliveau R (2002): The expression of rho proteins decreases with human brain tumor progression: potential tumor markers. Clin. Exp. Metastasis 19, 9-15 https://doi.org/10.1023/A:1013884426692

Harding MA, Theodorescu D (2007): RhoGDI2: a new metastasis suppressor gene: discovery and clinical translation. Urol. Oncol. 25, 401-406 https://doi.org/10.1016/j.urolonc.2007.05.006

Hinz B, Phan SH, Thannickal VJ, Galli A, Bochaton-Piallat M, Gabbiani G (2007): The myofibroblast: one function, multiple origins. Am. J. Pathol. 170, 1807-1816 https://doi.org/10.2353/ajpath.2007.070112

Jones MB, Krutzsch H, Shu H, Zhao Y, Liotta LA, Kohn EC, Petricoin EF (2002): Proteomic analysis and identification of new biomarkers and therapeutic targets for invasive ovarian cancer. Proteomics 2, 76-84

https://doi.org/10.1002/1615-9861(200201)2:1<76::AID-PROT76>3.0.CO;2-O

Ji H, Tang H, Lin H, Mao J, Gao L, Liu J, Wu T (2014): Rho/Rock cross-talks with transforming growth factor- $\beta / \mathrm{Smad}$ pathway participates in lung fibroblast-myofibroblast differentiation. Biomed. Rep. 2, 787-792

https://doi.org/10.3892/br.2014.323

Kishi T, Hirooka Y, Masumoto A, Ito K, Kimura Y, Inokuchi K, Tagawa T, Shimokawa H, Takeshita A, Sunagawa K (2005): Rho-kinase inhibitor improves increased vascular resistance and impaired vasodilation of the forearm in patients with heart failure. Circulation 111, 2741-2747 https://doi.org/10.1161/CIRCULATIONAHA.104.510248

Li G, Chen SJ, Oparil S, Chen YF, Thompson JA (2000): Direct in vivo evidence demonstrating neointimal migration of adventitial fibroblasts after balloon injury of rat carotid artery. Circulation 101, 1362-1365 https://doi.org/10.1161/01.CIR.101.12.1362

Lin X, Wen J, Liu R, Gao W, Qu B, Yu M (2018): Nintedanib inhibits TGF- $\beta$-induced myofibroblast transdifferentiation in human Tenon's fibroblasts. Mol. Vis. 24, 789-800

Pan P, Shen M, Yu H, Li Y, Li D, Hou T (2013): Advances in the development of Rho-associated protein kinase (ROCK) inhibitors. Drug. Discov. Today 18, 1323-1333 https://doi.org/10.1016/j.drudis.2013.09.010

Sartore S, Chiavegato A, Faggin E, Franch R, Puato M, Ausoni S, Pauletto P (2001): Contribution of adventitial fibroblasts to neointima formation and vascular remodeling: from innocent bystander to active participant. Circ. Res. 89, 1111-1121 https://doi.org/10.1161/hh2401.100844

Sun YB, Qu X, Caruana G, Li J (2016): The origin of renal fibroblasts/myofibroblasts and the signals that trigger fibrosis. Differentiation 92, 102-107 https://doi.org/10.1016/j.diff.2016.05.008

Tsai CC, Wu SB, Kau HC, Wei YH (2018): Essential role of connective tissue growth factor (CTGF) in transforming growth factor- $\beta 1$ (TGF- $\beta 1$ )-induced myofibroblast transdifferentiation from Graves` orbital fibroblasts. Sci. Rep. 8, 7276 https://doi.org/10.1038/s41598-018-25370-3

Tulis DA (2007): Rat carotid artery balloon injury model. Methods. Mol. Med. 139, 1-30 https://doi.org/10.1007/978-1-59745-571-8_1

Verrecchia F, Mauviel A (2002): Transforming growth factor- $\beta$ signaling through the Smad pathway: role in extracellular matrix gene expression and regulation. J. Invest. Dermatol. 118, 211-215 https://doi.org/10.1046/j.1523-1747.2002.01641.x

Wen J, Lin X, Gao W, Qu B, Ling Y, Liu R, Yu M (2019) MEK inhibition prevents TGF $\beta 1$ induced myofibroblast transdifferentiation in human tenon fibroblasts. Mol. Med. Rep. 19, 468-476 https://doi.org/10.3892/mmr.2018.9673

Wilcox JN, Scott NA (1997): Potential role of the adventitia in arteritis and atherosclerosis. Int. J. Cardiol. 54, (Suppl.), S21-S35 https://doi.org/10.1016/S0167-5273(96)02811-2

Zalewski A, Shi Y (1997): Vascular myofibroblasts: lessons from coronary repair and remodeling. Arterioscler. Thromb. Vasc. Biol. 17, 417-422 https://doi.org/10.1161/01.ATV.17.3.417

Zhao L, Wang H, Li J, Liu Y, Ding Y (2008): Overexpression of Rho GDP-dissociation inhibitor alpha is associated with tumor progression and poor prognosis of colorectal cancer. J. Proteome. Res. 7, 3994-4003 
https://doi.org/10.1021/pr800271b

Zhao L, Wang H, Sun X, Ding Y (2010): Comparative proteomic analysis identifies proteins associated with the development and progression of colorectal carcinoma. FEBS J. 277, 41954204

https://doi.org/10.1111/j.1742-4658.2010.07808.x

Zhang XY, Zhang T, Gao F, Li QL, Shen CY, Li YK, Li W, Zhang XM

(2015): Fasudil, a Rho-kinase inhibitor, prevents intima-media thickening in a partially ligated carotid artery mouse model: Effects of fasudil in flow-induced vascular remodeling. Mol. Med. Rep. 12, 7317-7325

https://doi.org/10.3892/mmr.2015.4409

Received: March 3, 2019

Final version accepted: May 7, 2019

First published online: June 20, 2019 